\title{
Efecto de la sustitución de V por Ti sobre las temperaturas de transformación de fase y el desajuste de red matriz/ precipitado en la superaleación 76Fe-12Al-12V
}

\author{
Effect of $V$ substitution by Ti on the phase \\ transition temperatures and matrix/ \\ precipitates lattice misfit of the \\ $76 \mathrm{Fe}-12 \mathrm{Al}-12 \mathrm{~V}$ superalloy
}

Pedro Antonio Ferreirós ${ }^{1,2}$, Paula Regina Alonso ${ }^{1}$, Daniel Roberto Vega ${ }^{1}$, Gerardo Héctor Rubiolo ${ }^{1,3}$

\footnotetext{
${ }^{1}$ Comisión Nacional de Energía Atómica - Centro Atómico Constituyentes (CNEA-CAC) e Instituto Sabato (UNSAMCNEA), Av. General Paz 1499, San Martín, Buenos Aires, Argentina e-mail: ferreiros@cnea.gov.ar,pralonso@cnea.gov.ar

${ }^{2}$ Universidad Tecnológica Nacional, Facultad Regional General Pacheco (UTN-FRGP), Av. Hipólito Yrigoyen 288 , Gral. Pacheco, Buenos Aires, Argentina

${ }^{3}$ Consejo Nacional de Investigaciones Científicas y Técnicas (CONICET), Av. Rivadavia 1917, Buenos Aires, Buenos Aires, Argentina

e-mail: rubiolo@cnea.gov.ar
}

\begin{abstract}
RESUMEN
Las aleaciones basadas en Fe-Al tienen un potencial considerable como materiales para aplicaciones estructurales en altas temperaturas. Sin embargo, su insuficiente resistencia a la termofluencia ha sido un obstáculo para su aplicación. La adición de un tercer aleante ( $\mathrm{Nb}, \mathrm{Ti}, \mathrm{Zr}$ ó Ta) ha conseguido aumentar su resistencia a altas temperaturas, pero a costa de una baja ductilidad. En trabajos previos y con el fin de resolver estas deficiencias, investigamos aleaciones ferríticas de $\mathrm{Fe}-\mathrm{Al}-\mathrm{V}$ con precipitación coherente de la fase $\mathrm{L}_{1}\left(\mathrm{Fe}_{2} \mathrm{AlV}\right)$ sobre una matriz A2. Entre las posibles aleaciones, por presentar precipitados L $2{ }_{1}$ de morfología esférica y sin efecto de coalescencia en alta temperatura, seleccionamos la superaleación $76 \mathrm{Fe}-12 \mathrm{Al}-12 \mathrm{~V}$. Buscamos ahora un cuarto aleante capaz de incrementar la temperatura de coexistencia del campo de dos fases A2+L2 y en consecuencia la máxima temperatura de aplicación. Encontramos que las secciones isotérmicas del rincón rico en Fe de los diagramas de fases ternarios Fe-Al-V y Fe-Al-Ti poseen campos de fases similares. Además, la relación entre las energías de formación calculadas para los intermetálicos L2 1 con Ti y V, permite predecir una temperatura de equilibrio mayor para el $\mathrm{Fe}_{2} \mathrm{TiAl}$ que para el $\mathrm{Fe}_{2} \mathrm{VAl}$. Por lo tanto, seleccionamos al Ti como posible $4^{\text {to }}$ aleante en la superaleación $76 \mathrm{Fe}-12 \mathrm{Al}-12 \mathrm{~V}$. En este trabajo mostramos que la sustitución del vanadio por titanio incrementa levemente la temperatura máxima de existencia del campo A2 + L2 $2_{1}$, además el desajuste de red matriz/precipitado es anulado para un contenido de Ti entre 0,5 y $1 \%$ atómico e incrementado en forma positiva a mayores porcentajes de Ti. Analizamos por otro lado la cinética de engrosamiento de los precipitados $\mathrm{L} 2{ }_{1}$ hallando que la velocidad aumenta con el agregado de Ti y la morfología se modifica de esférica a cúbica.
\end{abstract}

Palabras clave: Superaleación ferrítica, Transformaciones de fase, Desajuste de red.

\section{ABSTRACT}

Fe-Al based alloys have a remarkable potential for high temperature structural applications, provided that the limitation of their low creep resistance is solved. Third element addition $(\mathrm{Nb}, \mathrm{Ti}, \mathrm{Zr}$ or $\mathrm{Ta}$ ) has proven to perform the task, at the expense of a low ductility. In previous works we have investigated ferritic alloys in the 
Fe-Al-V system with coherent precipitation of the $\mathrm{L}_{1}$ phase $\left(\mathrm{Fe}_{2} \mathrm{AlV}\right)$ in the A2 matrix. Among possible alloys, we chose the $76 \mathrm{Fe}-12 \mathrm{Al}-12 \mathrm{~V}$ superalloy for filing a $\mathrm{L} 2{ }_{1}$ precipitation with spherical morphology and void coalescence at high temperature. The new task is to find a fourth alloy element in order to increase the temperature equilibrium of the two phases $\mathrm{A} 2+\mathrm{L} 22_{1}$ field and consequently the maximum application temperature. Isothermal sections of the Fe rich corner on ternary Fe-Al-V and Fe-Al-Ti phase diagrams have similar phase fields. Besides, by comparing the formation energies between $\mathrm{L}_{1}$ intermetallics of $\mathrm{Ti}$ and $\mathrm{V}$, it is expected a higher equilibrium temperature for the $\mathrm{Fe}_{2} \mathrm{TiAl}$ than for $\mathrm{Fe}_{2} \mathrm{VAl}$. Therefore we select titanium as a possible $4^{\text {th }}$ alloy element in the $76 \mathrm{Fe}-12 \mathrm{Al}-12 \mathrm{~V}$ superalloy We show in this work that vanadium substitution by titanium slightly increases the temperature limit for the $\mathrm{A} 2+\mathrm{L} 2$, phase field while cancelling the lattice misfit between matrix and precipitates for Ti content between 0.5 and 1 at. $\%$ and positively increasing it for Ti contents greater than 1 at. \%. Besides, we demonstrate that coarsening rate is increased with Ti addition and morphology is modified from spherical to cubic.

Keywords: Ferritic superalloy, Phase transformation, Lattice misfit.

\section{INTRODUCCIÓN}

Los aluminuros de hierro para aplicaciones de alta temperatura han atraído significativamente la atención de los investigadores durante varias décadas. Sus atractivas propiedades incluyen baja densidad, alta resistencia a la oxidación y bajo costo de materias primas [1-5]. Sin embargo, la reducida resistencia a la termofluencia por encima de los $600{ }^{\circ} \mathrm{C}$ para las aleaciones binarias $\mathrm{Fe}-\mathrm{Al}$ ha sido un obstáculo para su aplicación [6]. Para superar esta deficiencia sobre las fases intermetálicas, múltiples intentos fueron investigados utilizando terceros elementos de aleación. Sin embargo, este enfoque a menudo ha dejado al material con elevadas temperaturas de transición dúctil-frágil incluso cuando fue alcanzado el objetivo de incrementar la resistencia a la termofluencia [7-17]. El fortalecimiento de la solución sólida $\alpha$-Fe (A2) por partículas intermetálicas coherentes fue menos intentado y podría ser una posible solución $[18,19]$. En trabajos previos y con el fin de resolver estas deficiencias, investigamos aleaciones ferríticas de $\mathrm{Fe}-\mathrm{Al}-\mathrm{V}$ con precipitación coherente de la fase L2 ${ }_{1}\left(\mathrm{Fe}_{2} \mathrm{AlV}\right)$ sobre una matriz A2 $[19,20]$. Entre las posibles aleaciones, por presentar precipitados L2 ${ }_{1}$ de morfología esférica y sin efecto de coalescencia en alta temperatura, seleccionamos la superaleación $76 \mathrm{Fe}-$ $12 \mathrm{Al}-12 \mathrm{~V}[20,21]$. Buscamos ahora un cuarto aleante capaz de incrementar la temperatura de coexistencia del campo de dos fases $\mathrm{A} 2+\mathrm{L} 2{ }_{1}$ y en consecuencia la máxima temperatura de aplicación. Encontramos que las secciones isotérmicas del rincón rico en $\mathrm{Fe}$ de los diagramas de fases ternarios $\mathrm{Fe}-\mathrm{Al}-\mathrm{V}$ y Fe-Al-Ti poseen similares campos de fases. Además, la relación de las energías de formación calculadas para los intermetáli$\cos \mathrm{L} 2_{1}$ con $\mathrm{Ti}$ y $\mathrm{V}$, permiten predecir una temperatura de equilibrio mayor para el $\mathrm{Fe}_{2} \mathrm{TiAl}$ que para el $\mathrm{Fe}_{2} \mathrm{VAl}$ [19]. Por lo tanto seleccionamos al Ti como posible $4^{\text {to }}$ aleante en la superaleación $76 \mathrm{Fe}-12 \mathrm{Al}-12 \mathrm{~V}$ y estudiamos en este trabajo el efecto de la sustitución del vanadio por titanio sobre las temperaturas de transformación de fase.

Debido a que las propiedades mecánicas dependen fuertemente del desajuste de parámetro de red entre matriz y precipitado [22,23], es necesario estudiar paralelamente la influencia del agregado de Ti sobre este desajuste. Los valores reportados del parámetro de red del intermetálico de composición estequiométrica se encuentran en el intervalo: 0,5761-0,5774 nm [24-27]. La solución sólida de $\alpha$-Fe en la aleación $76 \mathrm{Fe}-12 \mathrm{Al}-$ $12 \mathrm{~V}$ posee según el diagrama de equilibrio de fases un contenido atómico de $\mathrm{Al}$ de hasta $9,4 \%$ a $700^{\circ} \mathrm{C}$ [20], y para dicho contenido de $9,4 \%$ at. en la fase $\alpha$-Fe puede estimarse un parámetro de red de $0,2882 \mathrm{~nm}$ a partir de la relación lineal obtenida en la Ref. [28] con una precisión de $10^{-4} \mathrm{~nm}$. Según estos valores reportados, el parámetro de red del precipitado $\mathrm{Fe}_{2} \mathrm{AlV}$ (estequiométrico) es 1,9996 veces el de la solución sólida A2 para la aleación $76 \mathrm{Fe}-12 \mathrm{Al}-12 \mathrm{~V}$, es decir prácticamente el doble. Debe ser aclarado que esta aproximación no tiene en cuenta factores como: el corrimiento de composición del intermetálico estequiométrico, el contenido de $\mathrm{V}$ en la fase matriz, la influencia de la temperatura, etc. Interesa entonces, conocer el efecto de la sustitución de $\mathrm{V}$ por Ti sobre el desajuste de red matriz/precipitado.

En este trabajo presentamos los resultados de mediciones de temperaturas de transformación de fases por el método de calorimetría diferencial de barrido y resultados de los parámetros de red de la matriz A2 y precipitado L2 1 por el método de difracción de rayos X, en seis aleaciones en las cuales se modifica progresivamente el contenido de Ti a expensas del de $\mathrm{V}$ hasta un máximo de $3 \%$ at.

\section{MATERIALES Y MÉTODOS}

Se fundieron aleaciones de aproximadamente $12 \mathrm{~g}$ en un horno de arco eléctrico con electrodo no consumible de $\mathrm{W}$, atmósfera de $\mathrm{Ar}$ y crisol de $\mathrm{Cu}$ refrigerado por agua. La pureza de los elementos utilizados fue 99,97\% Fe, 99,99\% Al, 99,7 \% V y 99,7 \% Ti. Las muestras envueltas en Ta y encapsuladas en un tubo de cuarzo con atmósfera de Ar fueron envejecidas térmicamente a $1100{ }^{\circ} \mathrm{C}$ por $136 \mathrm{~h}$. La composición de las aleaciones fue medida por microsonda electrónica con un equipo Cameca SXFive. Los valores con sus des- 
viaciones estándar fueron obtenidos del promedio de 50 mediciones puntuales a lo largo de una línea de 10 $\mathrm{mm}$. Las muestras destinadas a calorimetría diferencial de barrido (DSC) fueron cortadas por electroerosión en cilindros de $\emptyset 3 \times 3 \mathrm{~mm}$. Las temperaturas de transformación de fase se midieron en un equipo DSC LABSYS evo a $5{ }^{\circ} \mathrm{C} / \mathrm{min}$ y flujo de $\mathrm{Ar}$ de $4,2 \times 10^{-7} \mathrm{~m}^{3} / \mathrm{s}$.

Los resultados presentados se obtuvieron restándole una línea recta a la señal registrada, de forma tal que se anule la pendiente obtenida en la medición. La medición fue realizada en calentamiento, con un tratamiento previo de solubilización a $1100{ }^{\circ} \mathrm{C}$ por $900 \mathrm{~s}$ luego enfriamiento hasta $550{ }^{\circ} \mathrm{C}$ y finalmente la medición en calentamiento hasta alcanzar los $1100{ }^{\circ} \mathrm{C}$. Los tratamientos de envejecimiento se realizaron en el DSC luego del cíclo de medición de temperaturas de transformaciones de fases, enfriando desde $1100{ }^{\circ} \mathrm{C}$ a $90{ }^{\circ} \mathrm{C} / \mathrm{min}$ hasta $700{ }^{\circ} \mathrm{C}$ con un tiempo de permanencia de $1320 \mathrm{~s}$ y luego enfriando hasta temperatura ambiente. Las muestras envejecidas fueron observadas por microscopía electrónica de transmisión (TEM) en un Philips CM200 a 160kV. Se utilizó un porta muestras de doble inclinación, para observar las muestras en el plano $\{110\}$ y se tomaron campos oscuros en la dirección $\langle 111\rangle$ (estructura L $2_{1}$ ). Las láminas delgadas fueron cortadas por electroerosión, luego pulido con tela esmeril hasta espesores de $100 \mu \mathrm{m}$ y finalmente adelgazamiento electrolítico en un Tenupol-5 Struers, con electrolito formado por una parte de ácido nítrico y dos partes de metanol a $-30{ }^{\circ} \mathrm{C}$. Los polvos para mediciones de difracción de rayos-X (XRD) fueron obtenidos por limadura manual y tamizados en granulometría menor a $90 \mu \mathrm{m}$. El polvo fue tratado térmicamente (solubilizado a $900{ }^{\circ} \mathrm{C}$ por $900 \mathrm{~s}$ y envejecido a $700{ }^{\circ} \mathrm{C}$ por 1320 s) en un contenedor de Ta dentro de un tubo de cuarzo con atmósfera de vacío. Las mediciones de XRD fueron realizadas en un equipo Panalytical Empyrean con detector $\mathrm{PIXcel}^{3 \mathrm{D}}$ y radiación de $\mathrm{Cu} \mathrm{K} \alpha$. Dos condiciones de medición fueron utilizadas $20^{\circ}<2 \theta<120^{\circ}$ y $29^{\circ}<2 \theta<33^{\circ}$ en tiempos de 7200 s y pasos de $0,026^{\circ}$ en ambos casos. Los patrones de XRD fueron analizados por el método de refinamiento de Rietveld [29], el procedimiento de ajuste utilizado fue realizado con el programa FULLPROF [30] y WinPLOTR [31].

La secuencia iterativa de refinamiento aplicada para poder utilizar la información de dos difractogramas tomados con tiempos por paso diferentes se describe a continuación. Debe aclararse que el objetivo en el presente trabajo es hallar los parámetros de red de las dos fases presentes y que las simplificaciones adoptadas prácticamente no afectan a dichos resultados. El primer paso fue refinar el difractograma medido entre $20^{\circ}<2 \theta<120^{\circ}$, en esta operación se supuso una mezcla de fases de $21 \% \mathrm{~L} 2{ }_{1}$ y $79 \% \mathrm{~A} 2$ (\% peso) [20] y los parámetros de la fase $\mathrm{L} 2{ }_{1}$ se fijaron para obtener el tamaño de cristalita observado por TEM. Para las composiciones de las fases se utilizó el intermetálico estequiométrico $\mathrm{Fe}_{2} \mathrm{AlV}$ y $\alpha$-Fe puro. El perfil de pico utilizado fue del tipo Thompson-Cox-Hastings Pseudo-Voigt y el ancho instrumental se definió por una medición a iguales condiciones sobre un patrón de silicio. Luego, se refinó el difractograma de $29^{\circ}<2 \theta<33^{\circ}$ pero en este caso sólo con la fase L $2_{1}$ por contener únicamente su pico de difracción (200); en esta operación se impusieron los parámetros instrumentales y de forma del primer refinamiento, obteniendo como resultado del refinamiento el parámetro de red aproximado $\mathrm{a}_{\mathrm{L} 21} *$ de la fase $\mathrm{L} 2_{1}$.

La secuencia siguiente fue repetir el primer refinamiento de $20^{\circ}<2 \theta<120^{\circ}$ pero imponiendo ahora el resultado del parámetro de red $\mathrm{a}_{\mathrm{L} 21}$ *. Resultaron nuevos valores de parámetros instrumentales y de forma de la fase $\mathrm{L} 2{ }_{1}$, los cuales pudieron ser nuevamente refinados en el difractograma de $29^{\circ}<2 \theta<33^{\circ}$. Esta secuencia de refinamientos se repitió hasta la convergencia de los parámetros de red de las dos fases. El intervalo de confianza del parámetro de red fue obtenido configurando la fracción en volumen como variable en el procedimiento de refinamiento [32] y utilizando el mayor valor de corrimiento del parámetro de red de todos los patrones de XRD analizados y de forma independiente para cada fase (caso más desfavorable).

\section{RESULTADOS}

\subsection{Microestructura y transformaciones de fase}

Utilizando como punto de partida la aleación $76 \mathrm{Fe}-12 \mathrm{Al}-12 \mathrm{~V}$, se fabricaron 6 aleaciones en las cuales el contenido de titanio va incrementándose y reemplazando al porcentaje de vanadio hasta un máximo de 3 at.\%. La composición química medida en las aleaciones se presenta en la Tabla 1. Para observar los posibles cambios en la morfología de la precipitación $\mathrm{L} 2_{1}$ con la sustitución de pequeños porcentajes de $\mathrm{V}$ por $\mathrm{Ti}$, se seleccionó de nuestro trabajo previo [21] el tratamiento térmico de envejecimiento que produce el máximo endurecimiento en la aleación ternaria sin Ti. El envejecimiento seleccionado se realiza a $700{ }^{\circ} \mathrm{C}$ por $1320 \mathrm{~s}$ con un previo estadio en un campo monofásico de alta temperatura donde se disuelve la fase $\mathrm{L} 2{ }_{1}$, de esta forma el resultado obtenido debe ser una precipitación $\mathrm{L} 2_{1}$ de diámetro promedio $22 \mathrm{~nm}$ en una matriz A2. En la Figura 1 se muestran las imágenes de campo oscuro de TEM sobre las seis aleaciones con el tratamiento térmico de envejecimiento.

A partir de la sección del diagrama de equilibrio de fases presentado en [20], la aleación 76Fe-12Al$12 \mathrm{~V}$ debe presentar cuatro campos de fases, los cuales mencionados de menor a mayor temperatura son: 
$\left(\mathrm{A} 2+\mathrm{L} 2_{1}\right),\left(\mathrm{B} 2+\mathrm{L} 2_{1}\right), \mathrm{B} 2$ y A2. Las tres temperaturas de transformación de fase de las aleaciones fueron medidas por DSC y en la Figura 2 se presentan las mediciones obtenidas. La temperatura de trasformación de segundo orden A2/B2 se presenta como una elevación de la señal (marcada con una línea punteada sobre la Figura 2).

Tabla 1: Composición química medida por microsonda electrónica sobre aleaciones homogeneizadas térmicamente.

\begin{tabular}{c|c|c|c|c}
\hline ALEACIÓN & Fe (\%at.) & Al (\%at.) & V (\%at.) & TI (\%at.) \\
\hline 76Fe-12Al-12V & $75,83 \pm 0,18$ & $11,89 \pm 0,15$ & $12,28 \pm 0,13$ & - \\
\hline 76Fe-12Al-11,5V-0,5Ti & $75,63 \pm 0,20$ & $11,98 \pm 0,13$ & $11,90 \pm 0,14$ & $0,50 \pm 0,02$ \\
\hline 76Fe-12Al-11V-1Ti & $75,30 \pm 0,18$ & $12,42 \pm 0,12$ & $11,26 \pm 0,11$ & $1,02 \pm 0,02$ \\
\hline 76Fe-12Al-10,5V-1,5Ti & $75,33 \pm 0,20$ & $12,35 \pm 0,15$ & $10,76 \pm 0,13$ & $1,56 \pm 0,10$ \\
\hline 76Fe-12Al-10V-2Ti & $75,24 \pm 0,20$ & $12,38 \pm 0,16$ & $10,30 \pm 0,12$ & $2,07 \pm 0,04$ \\
\hline 76Fe-12Al-9V-3Ti & $75,25 \pm 0,14$ & $12,28 \pm 0,09$ & $9,33 \pm 0,10$ & $3,15 \pm 0,04$ \\
\hline
\end{tabular}
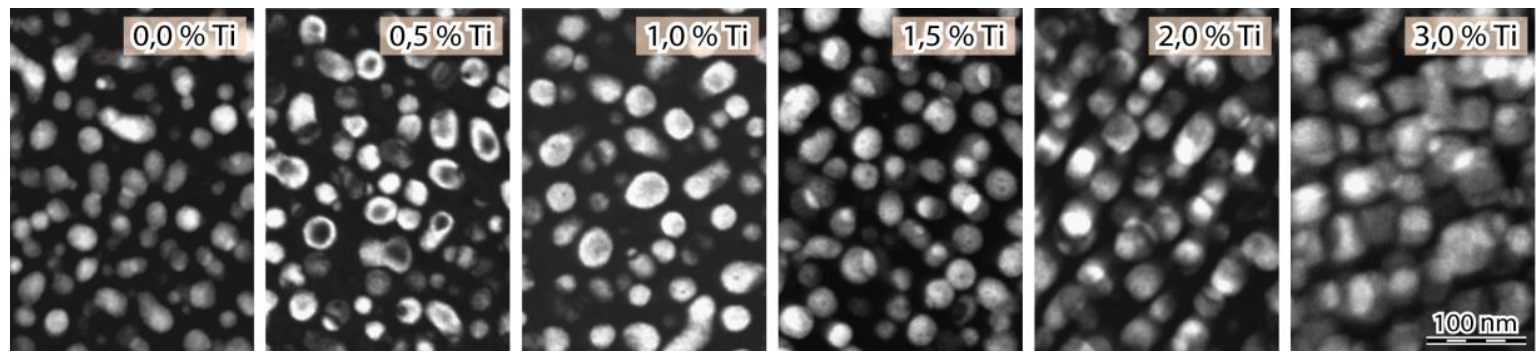

Figura 1: Imágenes TEM de campo oscuro mostrando los precipitados L2 (la matriz A2 se observa de color negro).

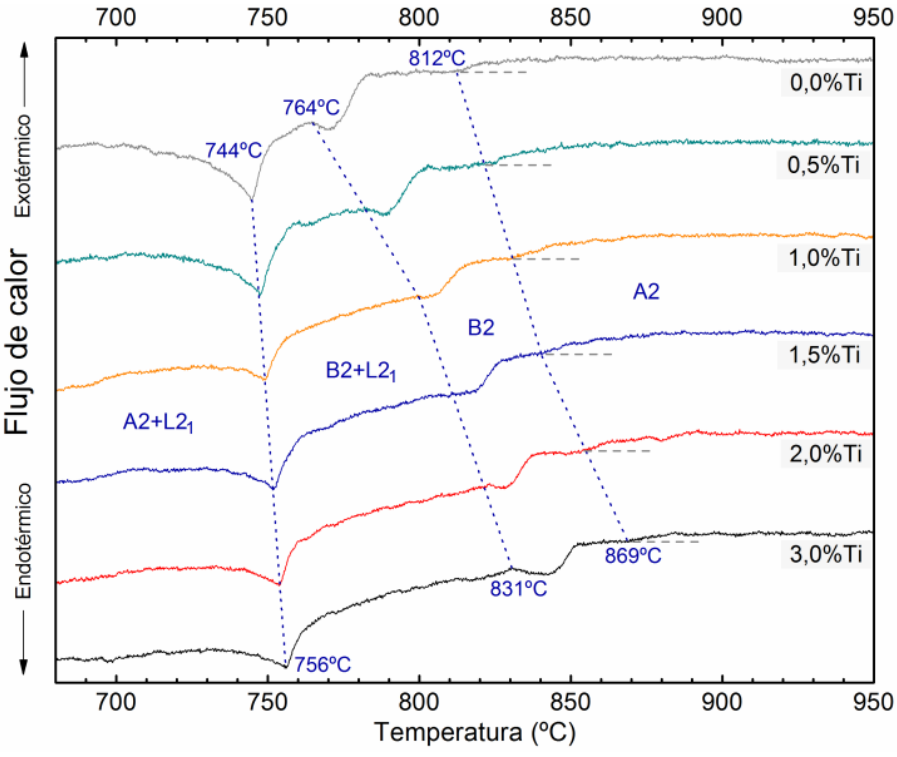

Figura 2: Medición por DSC en aleaciones $\mathrm{Fe}_{76} \mathrm{Al}_{12} \mathrm{~V}_{(12-\mathrm{x})} \mathrm{Ti}_{\mathrm{x}}$

\subsection{Desajuste de red matriz/precipitado}

Como se mencionara en la introducción, la fase precipitada L $2_{1}$ posee aproximadamente el doble del parámetro de red de la fase matriz A2. A continuación se presentan las mediciones experimentales de los parámetros de red de ambas fases para las 6 aleaciones realizadas.

La fase ordenada L $2_{1}$ es una super-red de la fase desordenada A2 y la gran similitud entre sus parámetros de red producen una superposición en sus patrones de XRD. El patrón de la fase L2 1 posee todos los picos de la A2 y los picos adicionales son de una intensidad menor, especialmente en las aleaciones de este trabajo donde la fase L2 $2_{1}$ tiene baja fracción en volumen y tamaño de cristalita nanométrico. Por lo tanto en el patrón de XRD con ambas fases presentes, los picos de mayor intensidad tienen el aporte de ambas fases y 
únicamente los picos de super-red de la $\mathrm{L} 2{ }_{1}$ se encuentran aislados pero con una muy baja intensidad. Debido a esta dificultad en la superposición de los patrones de XRD, se realizaron dos tipos de mediciones. La primera se realizó en un rango de $2 \theta$ amplio (Figura 3), donde se observan los picos de ambas fases superpuestos. La segunda medición se realiza en el entorno del pico (200) de la fase L $2_{1}$, adquiriendo una gran cantidad de cuentas hasta alcanzar distinguirlo claramente (420.00 cuentas aprox.), Figura 4. Por lo mencionado anteriormente, el proceso convencional de refinamiento por Rietveld para el caso en estudio es difícil de aplicar.

Para obtener los parámetros de red de las fases A2 y L2 ${ }_{1}$, se utilizó un procedimiento de refinamiento que considera los datos medidos en ambos difractogramas. El procedimiento fue detallado en la sección Materiales y Métodos. En la Figura 3a, se puede observar que las posiciones de Bragg de ambas fases son muy similares, en cambio para la aleación con $3 \%$ Ti de la Figura 3b, estas posiciones están más alejadas. En la ampliación del pico más intenso para la aleación sin Ti el pico (220)* se ubica a la derecha de la (110)' y con $3 \%$ Ti se invierte dicha ubicación. En la Figura 4, el pico (200) de la fase L $2_{1}$ es desplazado hacia la izquierda con el agregado de Ti, lo cual marca el aumento del parámetro de red al agregar Ti.
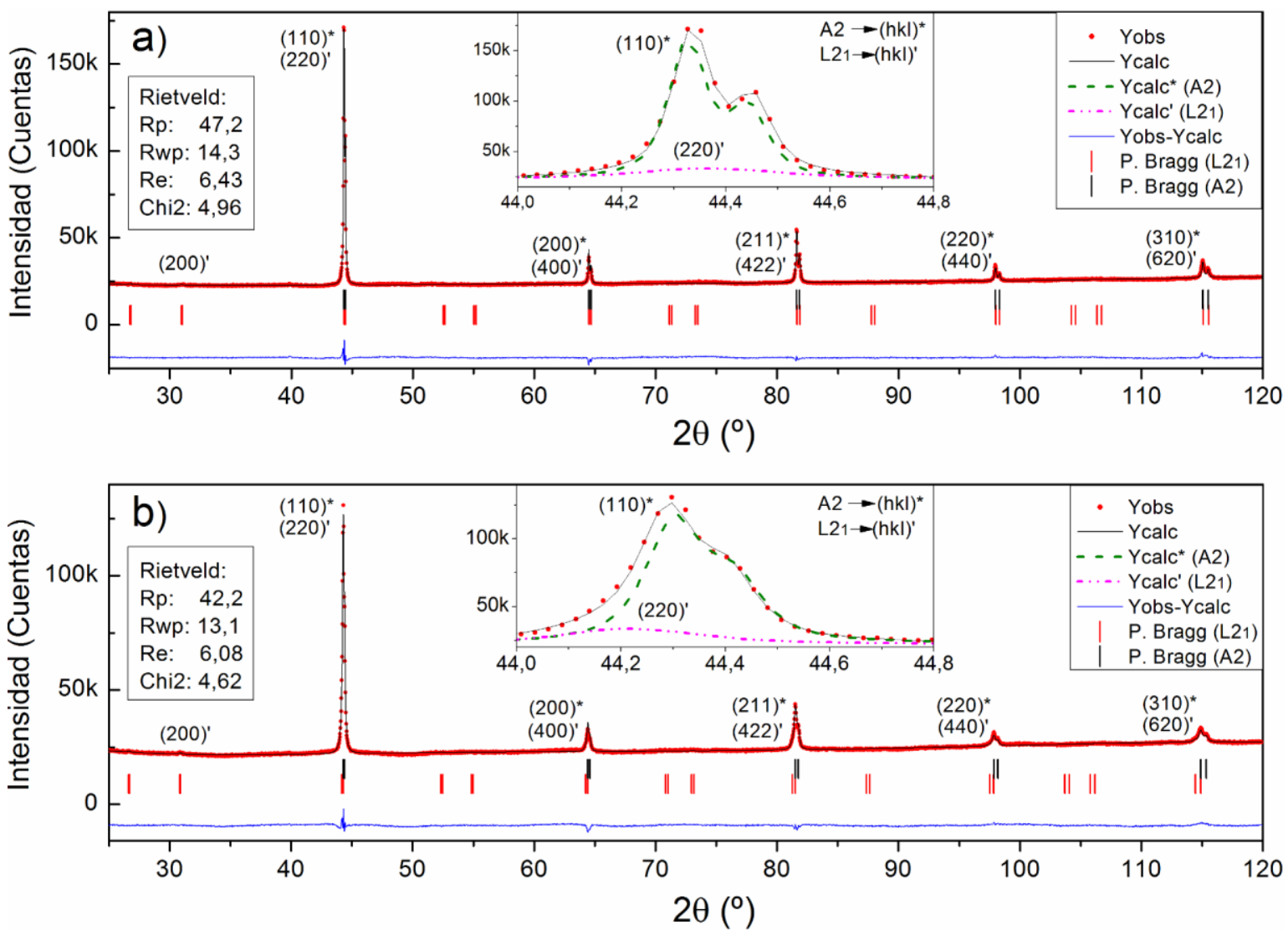

Figura 3: Patrones de XRD medidos (Yobs) y calculados (Ycalc) en aleaciones: a) 76Fe-12Al-12V, b) 76Fe-12Al-9V3Ti. El pico de mayor intensidad es ampliado y se grafica la contribución de ambas fases al patrón calculado (Ycalc $=$ Ycalc*+Ycalc'). 


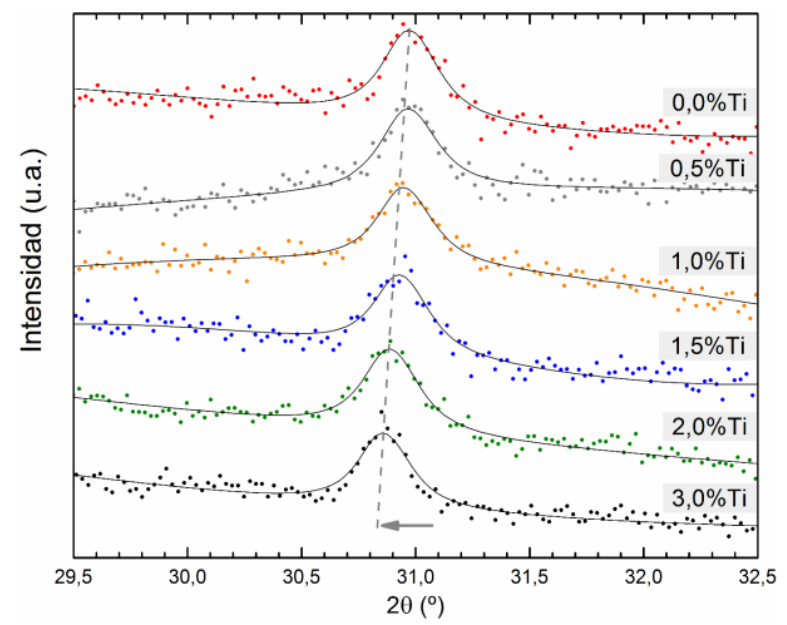

Figura 4: Patrones de XRD medidos y calculados en el pico (200) de la fase precipitada L $2{ }_{1}$.

En la Figura 5a se presentan los parámetros de red de las fases A2 y L2 1 obtenidos en el refinamiento. El desajuste entre los parámetros de red de ambas fases $(\delta)$ se define como [33]:

$$
\delta=\frac{2\left(a_{A}-a_{B}\right)}{a_{A}+a_{B}}
$$

Donde $a$ es el parámetro de red y el subíndice $A$ y $B$ indican las fases precipitada y matriz respectivamente. Sin embargo, en las aleaciones de este trabajo donde la relación de parámetros de red es de 1/2, la Ecuación 1 debe ser modificada de acuerdo a la siguiente expresión:

$$
\delta=\frac{2\left(a_{L 21}-2 a_{A 2}\right)}{a_{L 21}+2 a_{A 2}}
$$

El desajuste de red tratado en el presente trabajo corresponde al desajuste de red constreñido, es decir que difiere del obtenido en base a los parámetros de red de equilibrio de las fases individuales en estado relajado. En la Figura 5b se representa el porcentaje de desajuste de red entre las fases. En la Figura 5a se utilizó una relación de $1 / 2$ entre los ejes de ordenadas del gráfico, por lo tanto el cruce de las curvas en $\mathrm{x}=0,6 \% \mathrm{Ti}$, nos indica el punto donde $\delta$ se anula. Por encima o debajo de esta composición, las tensiones generadas por el $\delta$ cambian de signo. A su vez, se puede ver cómo el agregado de Ti incrementa más fuertemente el parámetro de red del precipitado $\mathrm{L} 2{ }_{1}$, aumentando el desajuste hasta el valor de $\delta=2.5 \times 10^{-3}$ para la aleación de $3 \% \mathrm{Ti}$. 


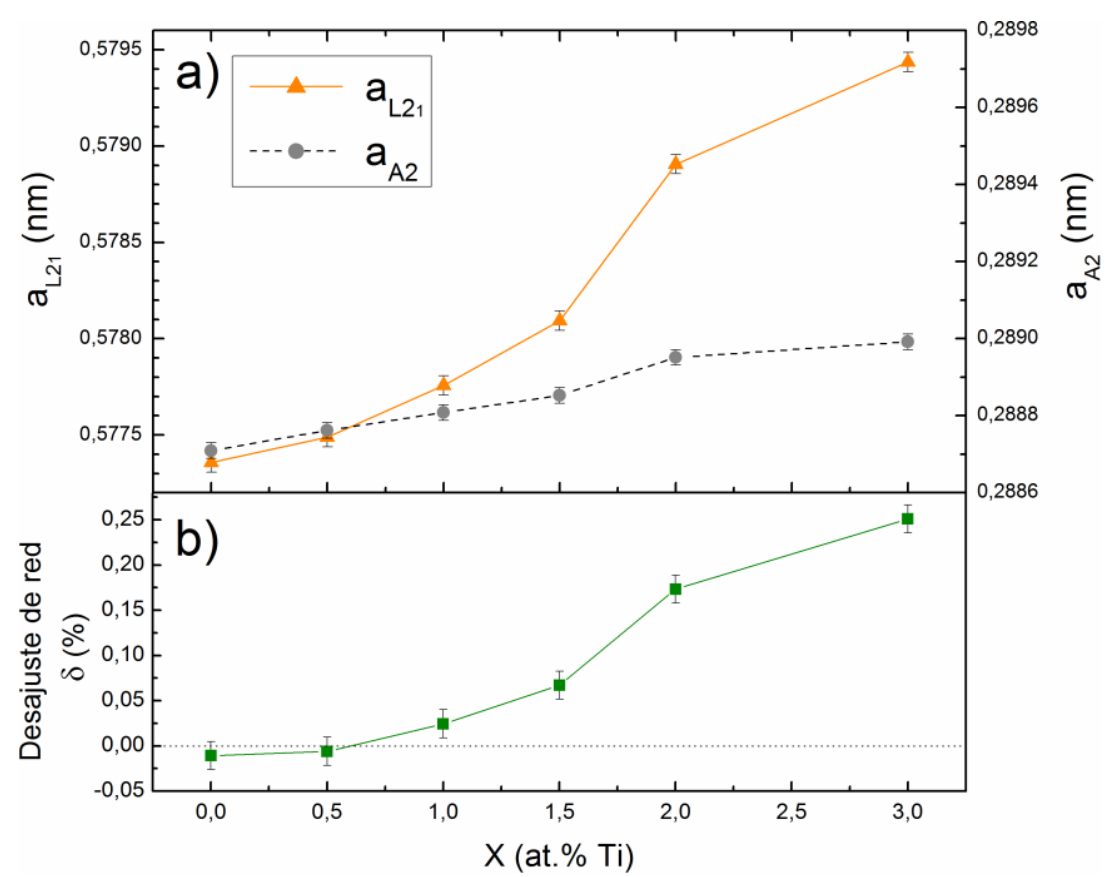

Figura 5: a) Parámetros de red de los precipitados $\mathrm{L} 2_{1}$ y la matriz $\mathrm{A} 2$. b) Desajuste de red en aleaciones $\mathrm{Fe}_{76} \mathrm{Al}_{12} \mathrm{~V}_{(12-}$ x) $\mathrm{Ti}_{\mathrm{x}}$.

\section{DISCUSIÓN}

Al observar la microestructura obtenida en las seis aleaciones a igual tiempo de tratamiento térmico de envejecimiento (Figura 1), puede deducirse que el reemplazo de V por Ti en la aleación 76Fe-12Al-12V produce un incremento en el tamaño de los precipitados. Esta observación cualitativa indica que la cinética de engrosamiento de los precipitados se ve incrementada a mayores contenidos de Ti. Adicionalmente puede notarse un pequeño cambio en la morfología de los precipitados L $2_{1}$. Al incrementar el contenido de Ti la morfología esférica tiende a ser cúbica. En trabajos anteriores con aleaciones ternarias Fe-Al-Ti fueron observados precipitados L2 ${ }_{1}$ de morfología cúbica sobre una matriz A2 [19,34], por lo tanto la pérdida progresiva de morfología esférica de precipitación al incrementar el contenido de Ti concuerda con la tendencia hallada.

En la Figura 6 se presentan las temperaturas de transformación de fase medidas en calentamiento por DSC (Figura 2) en función del porcentaje de Ti de las aleaciones. El efecto obtenido al reemplazar progresivamente el contenido de $\mathrm{V}$ por Ti es incrementar las tres temperaturas de transformación de fases, aunque para la transformación de menor temperatura el efecto es mucho más leve. Esto indica que el Ti, al menos hasta el contenido máximo utilizado (3\%at.), no es un elemento que permita ampliar de forma práctica el rango de aplicación de la superaleación hacia mayores temperaturas.

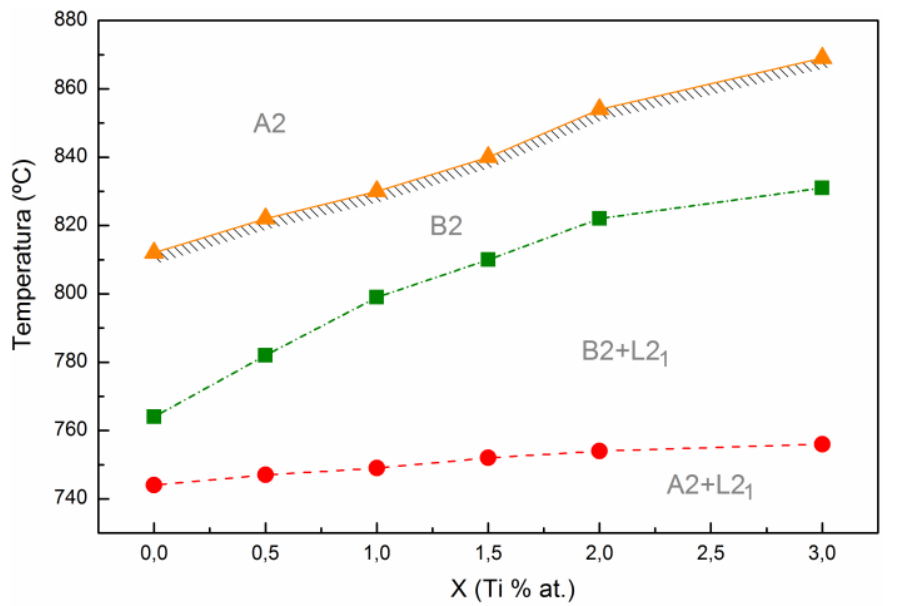

Figura 6: Transformaciones de fase medidas por DSC (Figura 2) en aleaciones $\mathrm{Fe}_{76} \mathrm{Al}_{12} \mathrm{~V}_{(12-\mathrm{x})} \mathrm{Ti}_{\mathrm{x}}$. La línea rayada representa la transformación de segundo orden.

Es conocido hace tiempo que el mayor desajuste de red promueve el incremento en la cinética de en- 
grosamiento de la precipitación [35] y la pérdida de la morfología esférica [36, 37]. Por lo tanto el aumento del $\delta$ obtenido en la Figura $5 \mathrm{~b}$ para contenidos de Ti altos, concuerda con la mayor velocidad de engrosamiento y tendencia a la morfología cúbica observadas en la Figura 1.

\section{CONCLUSIONES}

Las mediciones experimentales indican que los efectos producidos al reemplazar $\mathrm{V}$ por Ti en la aleación $76 \mathrm{Fe}-12 \mathrm{Al}-12 \mathrm{~V}$ son:

a. Incrementar las tres temperaturas de transformación de fases, especialmente las $\left(\mathrm{B} 2+\mathrm{L} 22_{1}\right) \rightarrow \mathrm{B} 2$ y $\mathrm{B} 2 \rightarrow \mathrm{A} 2$, y en menor medida la transformación de interés tecnológico $\left(\mathrm{A} 2+\mathrm{L} 2_{1}\right) \rightarrow\left(\mathrm{B} 2+\mathrm{L} 2_{1}\right)$.

b. Incrementar la velocidad de engrosamiento de los precipitados $\mathrm{L} 2{ }_{1}$.

c. Tendencia a modificar la morfología de precipitados de esférica a cúbica.

d. Anular el desajuste de red matriz/precipitado en contenidos de Ti entre 0,5 y $1 \%$ at.

e. Incrementar el desajuste de red de forma positiva por encima del $1 \% \mathrm{Ti}$.

Estos resultados son un aliciente para el estudio de las propiedades mecánicas de la aleación con agregado de $\mathrm{Ti}$, en particular del endurecimiento y los posibles mecanismos en función del incremento del desajuste de los parámetros de red entre matriz y precipitado.

\section{BIBLIOGRAFÍA}

[1] DEEVI, S.C., SIKKA V.K., "Nickel and iron aluminides: an overview on properties, processing, and applications", Intermetallics, v. 4, pp. 357-375, 1996.

[2] DEEVI, S.C., SIKKA, V.K., LIU, C.T., "Processing, properties, and applications of nickel and iron aluminides", Progress in Materials Science, v. 42, pp. 177-192, 1997.

[3] STOLOFF, N.S., "Iron aluminides: present status and future prospects", Materials Science and Engineering A, v. 258, pp. 1-14, 1998.

[4] MORRIS, D.G., MUÑOZ-MORRIS, M.A., "Recent developments toward the application of iron aluminides in fossil fuel technologies", Advanced Engineering Materials, v. 13, pp.43-47, 2011.

[5] MITRA, R., "Structural Intermetallics and Intermetallic Matrix Composites", London, CRC Press, 2015.

[6] LAWLEY, A., COLL, J.A., CAHN, R.W., "Influence of crystallographic order on creep of iron aluminum solid solutions", Transactions of the Metallurgical Society of AIME, v. 218, pp.166-76, 1960.

[7] EUMANN, M., PALM, M., SAUTHOFF, G., “Alloys based on $\mathrm{Fe}_{3} \mathrm{Al}$ or FeAl with strengthening $\mathrm{Mo}_{3} \mathrm{Al}$ precipitates", Intermetallics, v. 12, pp. 625-633, 2004.

[8] FALAT, L., SCHNEIDER, A., SAUTHOFF, G., et al., "Mechanical properties of Fe-Al-M-C (M=Ti, V, $\mathrm{Nb}, \mathrm{Ta}$ ) alloys with strengthening carbides and Laves phase", Intermetallics, v. 13, pp. 1256-1262, 2005.

[9] STEIN, F., PALM, M., SAUTHOFF, G., "Mechanical properties and oxidation behaviour of two-phase iron aluminium alloys with $\mathrm{Zr}(\mathrm{Fe}, \mathrm{Al})_{2}$ Laves phase or $\mathrm{Zr}(\mathrm{Fe}, \mathrm{Al})_{12} \tau_{1}$ phase", Intermetallics, v. 13, pp. 12751285,2005

[10] RISANTI, D.D., SAUTHOFF, G., "Strengthening of iron aluminide alloys by atomic ordering and Laves phase precipitation for high-temperature applications", Intermetallics, v. 13, pp. 1313-1321, 2005.

[11] SCHNEIDER, A., FALAT, L., SAUTHOFF, G., et al., "Microstructures and mechanical properties of $\mathrm{Fe}_{3} \mathrm{Al}$-based $\mathrm{Fe}-\mathrm{Al}-\mathrm{C}$ alloys”, Intermetallics, v. 13, pp. 1322-1331, 2005.

[12] KREIN, R., SCHNEIDER, A., SAUTHOFF, G., et al., "Microstructure and mechanical properties of $\mathrm{Fe}_{3} \mathrm{Al}$-based alloys with strengthening boride precipitates", Intermetallics, v. 15, pp. 1172-1182, 2005.

[13] KREIN, R., PALM, M., "The influence of $\mathrm{Cr}$ and $\mathrm{B}$ additions on the mechanical properties and oxidation behaviour of L2 $2_{1}$-ordered Fe-Al-Ti-based alloys at high temperatures", Acta Materialia, v. 56, pp. 24002405, 2008.

[14] RISANTI, D.D., SAUTHOFF, G., "Microstructures and mechanical properties of Fe-Al-Ta alloys with strengthening Laves phase”, Intermetallics, v. 19, pp. 1727-1736, 2011.

[15] MORRIS, D.G., REQUEJO, L.M., MUÑOZ-MORRIS, M.A., “A study of precipitation in $\mathrm{DO}_{3}$ ordered $\mathrm{Fe}-\mathrm{Al}-\mathrm{Nb}$ alloy", Intermetallics, v. 13, pp. 862-871, 2005.

[16] MORRIS, D.G., MUÑOZ-MORRIS, M.A., "Room and high temperature deformation behaviour of a forged $\mathrm{Fe}-15 \mathrm{Al}-5 \mathrm{Nb}$ alloy with a reinforcing dispersion of equiaxed Laves phase particles" Materials Science and Engineering A, v. 552, pp. 134-144, 2012. 
[17] KREIN, R., PALM, M., "Two-fold flow stress anomaly in L2 ${ }_{1}$-ordered Fe-Al-Ti-based alloys", Materials Science and Engineering A, v. 460-461, pp. 174-179, 2007.

[18] KREIN, R., PALM, M., HEILMAIER, M., "Characterization of microestructures, mechanical properties, and oxidation behavior of coherent A2+L2 ${ }_{1}$ Fe-Al-Ti.", Journal of Materials Research, v. 24, pp. 3412-3421, 2009.

[19] ALONSO, P.R., GARGANO, P.H., BOZZANO, P.B., et al., Intermetallics, v. 19, pp. 1157-1167, 2011.

[20] FERREIRÓS, P.A., ALONSO, P.R., GARGANO, P.H., et al., "Characterization of microstructures and age hardening of Fe1-2xAlxVx alloys", Intermetallics, v. 50, pp. 65-78, 2014.

[21] FERREIRÓS, P.A., ALONSO, P.R., RUBIOLO, G.H., "Coarsening process and precipitation hardening in $\mathrm{Fe}_{2} \mathrm{AlV}$-strengthened ferritic $\mathrm{Fe}_{76} \mathrm{Al}_{12} \mathrm{~V}_{12}$ alloy", Materials Science and Engineering A, v. 684, pp. 394-405, 2017.

[22] MUGHRABI, H., "Microstructural aspects of high temperature deformation of monocrystalline nickel base superalloys: some open problems", Materials Science and Technology, v. 25, pp. 191-204, 2009.

[23] ZENK, C.H., NEUMEIER, S., STONE, H.J., et al., "Mechanical properties and lattice misfit of $\gamma / \gamma$ ' strengthened Co-base superalloys in the Co-W-Al-Ti quaternary system", Intermetallics, v. 55, pp. 28-39, 2014.

[24] NISHINO, Y., "Unusual electron transport in Heusler-type $\mathrm{Fe}_{2} \mathrm{VAl}$ compound", Intermetallics, v. 8, pp. 1233-1241, 2000.

[25] KAWAHARADA, Y., KUROSAKI, K., YAMANAKA, S. J., "Thermophysical properties of $\mathrm{Fe}_{2}$ VAl", Journal of Alloys and Compounds, v. 352, pp. 48-51, 2003.

[26] VASUNDHARA, M., SRINIVAS, V., RAO, V,V., "Evidence for cluster glass behavior in $\mathrm{Fe}_{2} \mathrm{VAl} \mathrm{Heu-}$ sler alloys", Physical Review B, v. 78, 064401, pp. 1-10, 2008.

[27] SATO, K., NAKA, T., TAGUCHI, M., et al., "Weak itinerant ferromagnetism in Heusler-type $\mathrm{Fe}_{2} \mathrm{VAl}_{0.95}$ ", Physical Review B, v. 82, 104408, pp. 1-8, 2010.

[28] TAYLOR, A., JONES, R.M., "Constitution and magnetic properties of iron-rich iron-aluminum alloys", Journal of Physics and Chemistry of Solids, v. 6, pp. 16-37, 1958.

[29] RIETVELD, H.M., "A profile refinement method for nuclear and magnetic structures", Journal of Applied Crystallography, v. 2, pp. 65-71, 1969.

[30] RODRIGUEZ-CARBAJAL, J., "Recent advances in magnetic structure determination by neutron powder diffraction" Physica B: Condensed Matter, v. 192, pp. 55-69, 1993.

[31] ROISNEL, T., RODRÍGUEZ-CARVAJAL, J., "WinPLOTR: a Windows tool for powder diffraction patterns analysis", Materials Science Forum, v. 378-381, pp. 118-123, 2001.

[32] COLLINS, D.M., YAN, L., MARQUIS, E.A., et al., "Lattice misfit during ageing of polycrystalline nickel-base superalloy", Acta Materialia, v. 61, pp. 7791-7804, 2013.

[33] REED, R.C., RAE, C.M.F., "Physical Metallurgy of the Nickel-Based Superalloys", In: Laughlin, D.E., Hono, K., (eds), Physical Metallurgy, 5 ed., chapter 22, Vol. III, Elsevier, 2014.

[34] MENDIRATTA, M.G., EHLERS, S.K., LIPSITT, H.A., " $\mathrm{DO}_{3}-\mathrm{B} 2-\alpha$ Phase Relations in Fe-Al-Ti Alloys", Metallurgical Transactions A, v. 18, pp. 509-518, 1987.

[35] MUGHRABI, H., "The importance of sign and magnitude of $\gamma / \gamma$ ' lattice misfit in superalloys-with special reference to the new $\gamma$ '-hardened cobalt-base superalloys", Acta Materialia, v.81, pp. 21-29, 2014.

[36] RICKS, R.A., PORTER, A.J., ECOB, R.C., "The growth of $\gamma$ ' precipitates in nickel-base superalloys", Acta Metallurgica, v. 31, pp. 43-53, 1983.

[37] ROYER, A., BASTIE, P., BELLET, D., et al., "Temperature dependence of the lattice mismatch of the Am1 superalloy influence of the $\gamma$ 'precipitates' morphology", Philosophical Magazine A, v. 72, pp. 669-689, 1995. 